\title{
Biodegradation of organophosphorus insecticide methyl parathion by soil microorganisms
}

\author{
Thi Hue $\mathrm{Le}^{1^{*}}$, Quang Cuong Hoang ${ }^{1}$, Dinh Duy $\mathrm{Vu}^{2}$ and Thi Hoai Thu $\mathrm{Vo}^{1}$ \\ ${ }^{1}$ Institute of biotechnology, Vietnam - Russia Tropical Center, 63 Nguyen Van Huyen Str., Ha Noi, \\ Vietnam \\ ${ }^{2}$ Institute of Tropical Ecology, Vietnam - Russia Tropical Center, 63 Nguyen Van Huyen Str., Ha \\ Noi, Vietnam
}

\begin{abstract}
Organophosphorus compounds (OPs) have been widely used as effective insecticides. As a result, using too many OPs causes the residues of pesticides to be washed away into the water or soil, not only polluting water and soil, also directly or indirectly affecting environment and human health. Besides many countries and terrorists also use them as chemical warfare weapons. They are very dangerous neurotoxins to humans, animals and the environment. In the soil, there are many microorganisms that can degrade OPs, helping to release the accumulation of these toxic substances. In this study, two effective OP-degrading bacterial strains P9 and H14 has been isolated from agricultural land in Ha Nam province, Vietnam. We had used DNA barcodes (16S rRNA) to molecular identification of Klebsiella variicola (P9) and Priestia aryabhattai (H14) and deposited in GenBank MW644772, MW644771, respectively. Moreover, at an initial concentration of methyl parathion $50 \mathrm{mg} / \mathrm{L}$, in investigative culture mediums and conditions, strain P9 degraded $100 \%$ of methyl parathion after 7 days in Luria-Bertani (LB) liquid culture. Maximum growth of P9 strains was observered after 6 days incubation (OD600 $=3.34)$. This study is an effort to open a direction of applying them in the treatment of OPs contaminated soils and water.
\end{abstract}

\section{Introduction}

Organophosphates (OPs) are a group of phosphorus-containing organic compound that are esters of phosphoric acid. OPs inactivate a large enzyme family of the serine hydrolases group (Lipase and Esterase), including a very important enzyme - Acetylcholinesterase (AchE). AChE is an enzyme that hydrolyzes the neurotransmitter acetylcholine, which is involved in the termination of impulse transmission between nerve cells. The acute toxicity of OPs is related to irreversible inactivation of $\mathrm{AChE}$, prevents $\mathrm{AChE}$ from breaking down acetylcholine, leading to acetylcholine accumulation, disrupting nerve impulse transmission, causing seizures, muscle weakness, bradycardia, and respiratory failure leading to death [1]. These compounds are very toxic to humans, animals and the

Corresponding author: huele581985@gmail.com 
environment but have been widely used as effective insecticides (methyl parathion- MP, chlorpyrifos, diazinon, DFP...). In addition, German and British scientists synthesized OP neurotoxins that classified as Chemical Warfare Agents (CWA) (tabun, sarin, soman, $\mathrm{VX}, .$.$) . These are extremely toxic substances, can be released in liquid or gas form and$ easily kill a victim within only a few minutes and were used in battles and terrorist attacks that killed many people [2].

Degradation of OPs is a process that includes biodegradation, chemical hydrolysis and the effects of some physicochemical factors. In which, biodegradation is the main mechanism of the degradation and detoxification of pesticides in the soil. Soil microorganisms help to degrade OPs efficiently, safely and cheaply, so they have become the focus of many studies. They belonging to genera Aspergillus, Cladosporium, Penicillium, Flavobacterium, Pseudomonas, Bacillus, Lactobacillus, Serratia, Stenotrophomonas, Burkholderia, Athrobacter, Plesiomonas, etc [3]. Some soil microorganisms that degrade many types of OPs have been found: Flavobacterium sp. (degrades diazinone and parathion) [4], P. aeruginosa (acephate, methamidophos, MP, dimethoate, malathion) [5]. S. marcescens (chlorpyrifos, fenitrothion, and parathion) [6]. Stenotrophomonas sp. G1 (MP, methyl paraoxon, diazinon, phoxim, parathion, chlorpyrifos, profenofos, triazophos); Pseudomonas sp. WBC-3 (MP, chlorpyrifos, ethyl parathion and sumithion) [7]. Mansee et al published: Flavobacterum sp. (ATCC 27551), P. diminuta $\mathrm{MG}$, Alteromonas sp. JD6.5.19,... hydrolyze strongly many OP insecticides and also the CWA: Sarin, Soman [8],...

The specific objective of this study was isolated and characterize soil bacteria strains that can degrade OP pesticides and to select suitable media for good OP degradation.

\section{Materials and Methods}

\subsection{Media and chemicals}

All the chemicals used were of analytical grade: Methyl parathion $(99 \%)$ and Diazinon (99\%) (Sigma-Aldrich). The medium used in the study: mineral medium (MM, pH 7.0) [9], Wakimoto medium [10]; Luria-Bertani medium (LB, pH7) (g/L: Tryptone $10 \mathrm{~g}$; Yeast Extract $5 \mathrm{~g} ; \mathrm{NaCl} 10 \mathrm{~g}$ ). Soil samples were collected from agricultural land that was frequently sprayed with pesticides in Hanam, Vietnam and stored at $4{ }^{\circ} \mathrm{C}$ before being studied in laboratory.

\subsection{Methods}

\subsubsection{Enrichment and isolation of bacterial strains}

A total of $15 \mathrm{~g}$ soil sample was suspended in $50 \mathrm{ml}$ of minimal medium containing $50 \mathrm{mg} / \mathrm{l}$ of methyl parathion in a $250-\mathrm{ml}$ flask and incubated at $30^{\circ} \mathrm{C}$ on a shaker for 7 days. The incubation flask was allowed to settle for 30 minutes. $2 \mathrm{ml}$ suspension was then used to inoculate a fresh $30 \mathrm{ml}$ of minimal medium containing $30 \mathrm{mg} \mathrm{MP}$ and incubated at $30^{\circ} \mathrm{C}$ on a shaker for 7 days. After several similar transfers during which the MP concentration was gradually raised to $500 \mathrm{mg} / \mathrm{l}$. At the last transfer, an aliquot of culture solution was sampled, diluted and spread on the MP-minimal medium agar plates containing $200 \mathrm{mg} / \mathrm{L}$ of MP and incubated for 3 days at $30^{\circ} \mathrm{C}$. After incubation, single colonies were picked for further plate cultivation until single colonies of uniform morphology were obtained. 


\subsubsection{Determination of pesticide degradation}

The isolates were transferred into $\mathrm{MM}$ medium and incubated at $30{ }^{\circ} \mathrm{C}$ on a rotary shaker at $200 \mathrm{r} / \mathrm{min}$. After 16-18 h incubation, cells were harvested by centrifugation for $10 \mathrm{~min}$ at $4000 \mathrm{r} / \mathrm{min}$ and $4^{\circ} \mathrm{C}$, washed three times with sterile distilled water and finally resuspended in sterile distilled water. The cell suspension was then inoculated into MM with MP or Diazinon at $50 \mathrm{mg} / \mathrm{L}$ and OD $600 \mathrm{~nm}=0.7$ and incubated in the dark on a rotary shaker at $150 \mathrm{r} / \mathrm{min}$, and samples were taken at specific time intervals $(0,2,4,6,8,10,12,14$ days) for analysis.

\subsubsection{Thin layer chromatography (TLC)}

Culture solutions were extracted in a solvent mixture Toluene: Acetone (2:1) and TLC with hexane-acetone (4: 1) mobile phase [11]. After air drying in a hood, MP was determined by uv $254 \mathrm{~nm}$.

\subsubsection{Gas Chromatography/Mass Spectrometer (GC/MS)}

Culture samples were diluted with acetonitril, mix, centrifuged at $10,000 \times \mathrm{g}$ for $10 \mathrm{~min}$ to remove bacterial cells and passed through a $0.22-\mu \mathrm{m}$ filter. The collected supernatant was used as input for determination in the Thermo Scientific ${ }^{\mathrm{TM}}$ ISQ $^{\mathrm{TM}} 7000$ Single Quadrupole GC-MS system. Data handling and system operations were controlled by the Chromeleon 7 software. Separation was carried out using a TraceGOLD TG-5SilMS column (30 m x $0.25 \mathrm{~mm}, 0.25 \mu \mathrm{m}$, contained $5 \%$ phenylmethylpolysiloxane). Heli was used as the carier gas at a constant flow rate of $1.5 \mathrm{ml} / \mathrm{min}$. Sample injection volume was $1.0 \mu \mathrm{l}$. Injector temperature was kept at $230^{\circ} \mathrm{C}$ in splitless mode $(0.5 \mathrm{~min})$ and split flow $100 \mathrm{~mL} / \mathrm{min}$. The temperature of column was programmed at an initial temperature of $40{ }^{\circ} \mathrm{C}$ for $1.5 \mathrm{~min}$, raised to $180{ }^{\circ} \mathrm{C}$ at a rate of $25^{\circ} \mathrm{C} / \mathrm{min}$ and then raised to $280{ }^{\circ} \mathrm{C}$ at a rate of $5{ }^{\circ} \mathrm{C} / \mathrm{min}$ and then raised to $300^{\circ} \mathrm{C}$ at a rate $10{ }^{\circ} \mathrm{C} / \mathrm{min}$ and held for $5 \mathrm{~min}$.

The MS ionization was carried out in the electron ionization mode. The spectra were obtained at $70 \mathrm{eV}$. The MS transfer line and the ionization source temperature were set at 280 and $200^{\circ} \mathrm{C}$, respectively.)

\subsubsection{Molecular and biochemical characterization}

The selected isolate was selected isolate was biochemically characterized by Gram's reaction, carbohydrate fermentation (utilization of carbon sources), and some biochemicall test. It was also identified by $16 \mathrm{~S}$ rDNA gene sequence analysis after its $16 \mathrm{~S}$ rRNA gene fragment was amplified by colony polymerase chain reaction (PCR) using the primers $27 \mathrm{~F}$ (5'-AGAGTTTGATCCTGGCTCAG-3') and 1492R (5'-GGTTACCTTGTTACGACTT3') [12]. The 16S rDNA nucleotide sequence has been compared with the nucleotide sequence data on the Gene Bank (https://blast.ncbi.nlm.nih.gov/Blast.cgi). 


\section{Results and Discussion}

\subsection{Isolation of MP degrading microrganisms}

\subsubsection{Isolation and evaluation of the growth and degradation of OPs}

After isolation, 6 strains with different colony morphology (N1, H3, H14, P9, K3, K7) were selected on MM agar containing MP $200 \mathrm{mg} / \mathrm{L}$. They were cultured in MM broth containing MP $50 \mathrm{mg} / \mathrm{l}$ and MM containing Diazinon $50 \mathrm{mg} / \mathrm{l}$ on 14 days for preliminary assessment of OPs reducing property by TLC (Fig. 1) and for growth ability on MM plates supplemented with MP or Diazinon $300 \mathrm{mg} / \mathrm{l}$.

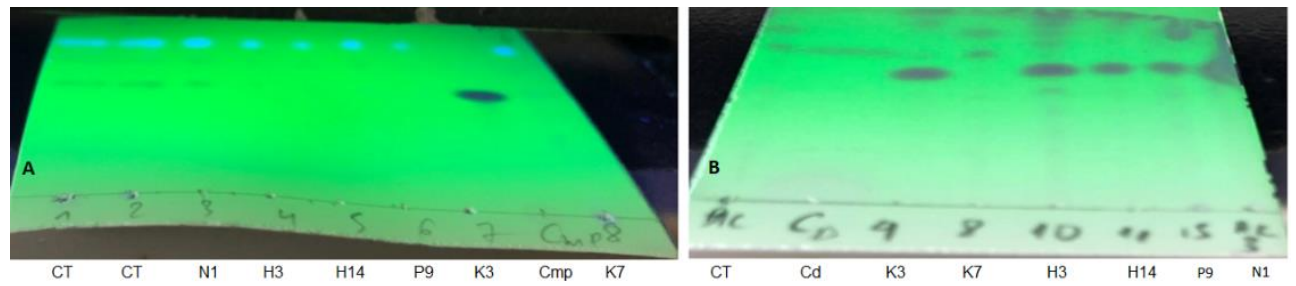

Fig. 1. Six isolates were cultured in the MM supplemented with MP (A) and Diazinon (B): CT (control: MM supplemented with OP but without strain), Cmp (mp Standard), Cd (Diazinon Standard)

A total of five strains $(\mathrm{H} 3, \mathrm{H} 14, \mathrm{P} 9, \mathrm{~K} 3, \mathrm{~K} 7)$ can degrade MP, 5 strains (K3, H3, H14, P9, N1) can degraded Diazinon and 2 strains (H14 and P9) that degraded best both MP and Diazinon after 14 days of incubation. However, P9 had better growth ability on both MM plate containing MP $300 \mathrm{mg} / \mathrm{l}$ and MM with Diazinon $300 \mathrm{mg} / 1$. P9 colony size was about 5-6 mm after 7 days of incubation while H14 was 3-4 mm. Thus, P9 was selected for further studies.

\subsubsection{Taxonomic characterization}

On LB agar plate after 3 days of incubation, colonies of P9 appeared in circular form, 3-4 $\mathrm{mm}$ in diameter, convex, shadow, slimy moist, creamy white (Fig. 2A). Under microscope, the bacterium appeared with short rod shape, 0.5-0.8 $\mu \mathrm{m}$ (Fig. 2B).
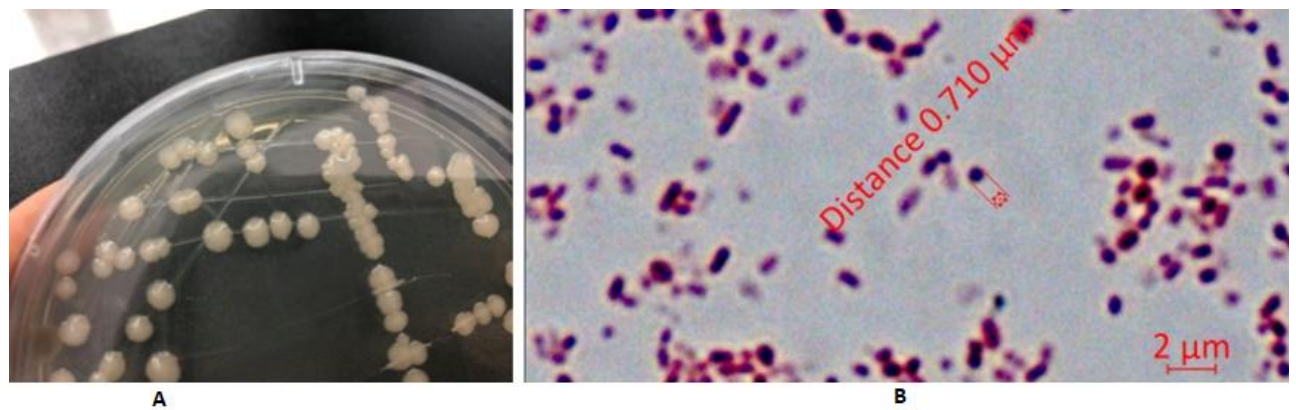

Fig. 2. Colony morphology on LB agar (image A) and microscopic morphology (image B) of bacterial strain P9

P9 was Gram-negative, non-motile bacillus, aerobic and can use various carbon sources such as sucrose, glucose, maltose, mannitol, rhamnose but can't use lactose, adonitol and 
citrate. Strain P9 was positive for ONPG, Urease but negative for indole, $\beta$-gluonidase, $\alpha$ galactosidase. In addition to morphological and biochemical characteristics, strains were also identified genetically through the $16 \mathrm{~S}$ rRNA sequence.

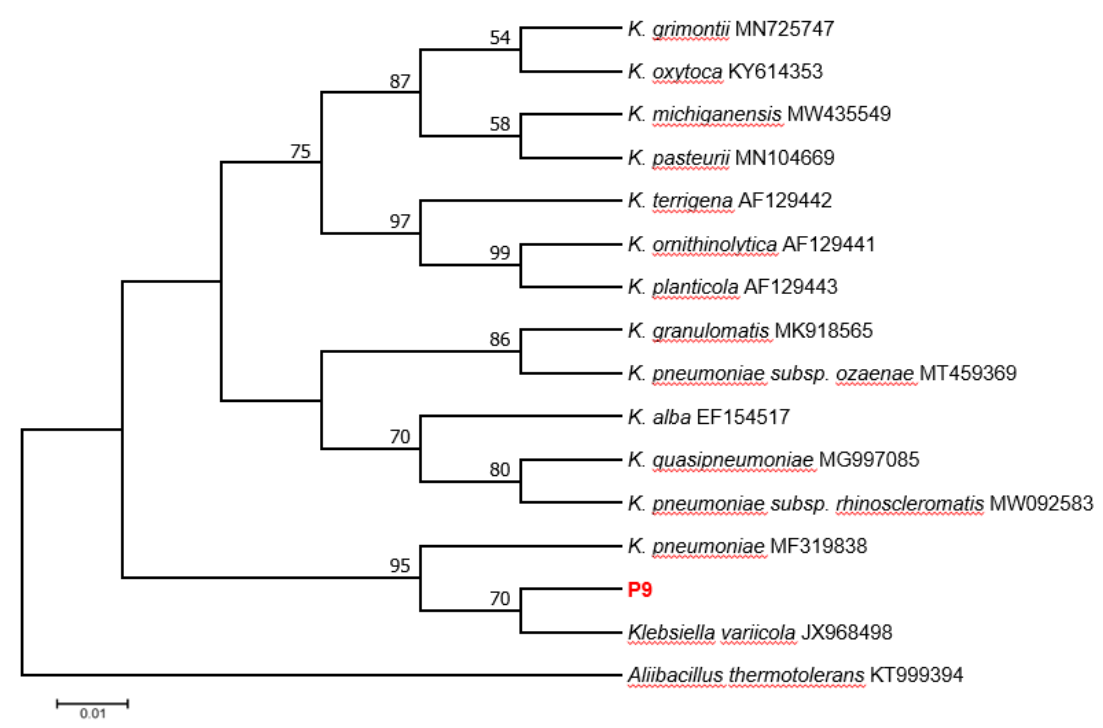

Fig. 3. Phylogenetic tree constructed from $16 \mathrm{~S}$ partial rRNA gene sequences of strain P9 using the Maximum likelihood (ML) tree

The partial 16S rRNA of bacterial strains P9 and H14 were sequenced and deposited in the GenBank with accession number MW644772 and MW644771, respectively. The gene was analyzed and aligned with corresponding genes in GenBank to create the phylogenetic tree as described in Figure 3. The isolate P9 has the most identity to Klebsiella variicola JX968498 (with $99.93 \%$ similarity in 16S rRNA gene sequences) (Fig. 3). Combined with the results of analysis of morphological and biochemical characteristics, the results of identification by sequencing are completely reliable. Klebsiella able to degrade OPs has been published by Kulkarni et al:: Klebsiella pneumoniae sp. S3 from the soil degraded tributyl phosphate [13], Kumar et al.: Klebsiella sp. degraded chlorpyrifos [3].

The isolate H14 has the most identity to Priestia aryabhattai MT605509 (identity of 100\%) (Fig. 4). This result is similar to the research result of Pailan et al:: Bacillus aryabhattai (Priestia aryabhattai) can degrade OPs (chlorpyrifos and parathion) well [12]. 


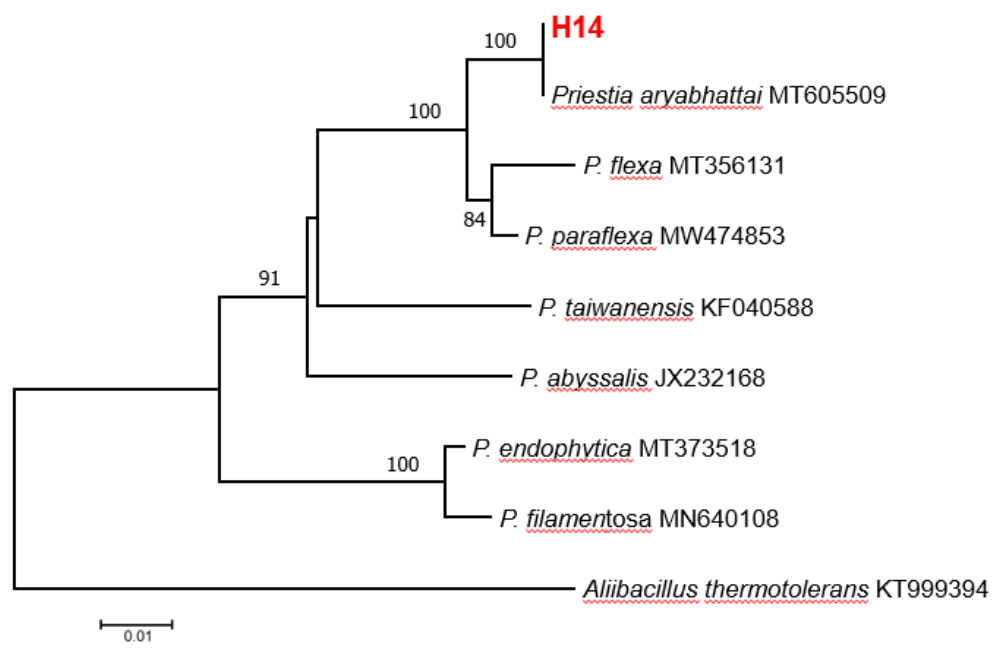

Fig. 4. Phylogenetic tree constructed from $16 \mathrm{~S}$ partial rRNA gene sequences of strain H14 using the Maximum likelihood (ML) tree

\subsection{Effect of the culture medium and conditions on the growth and MP degradation}

\subsubsection{MP reducing on MM medium}

The growth of bacterial strain P9 determined through OD600 and the degradation kinetic of MP in liquid MM medium in dependence to culture time was evaluated by GC / MS method (Fig. 5).

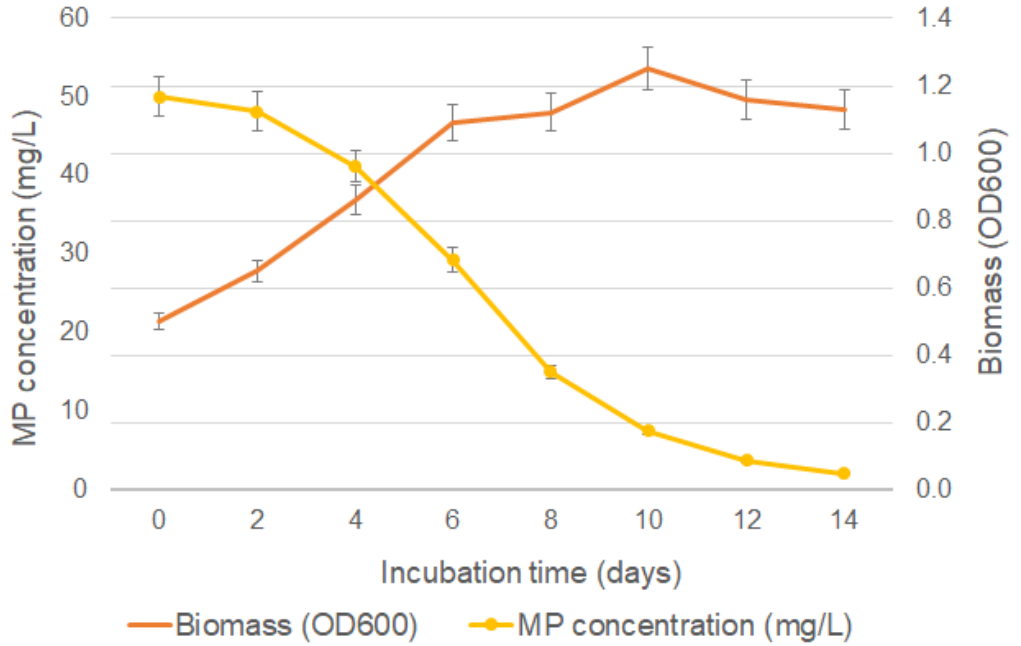

Fig. 5. The growth and MP degradation of bacterial strain P9

As shown Figure 5 indicated significant differences in growth kinetics of P9 in liquid culture within 14 days. The bacterial culture attained a maximum OD600 after 10 days $(\mathrm{OD} 600=1.25)$. The results showed that after the first 2 days of slow MP degradation, the 
strain began to degrade MP strongly. After 4, 6, 14 days of incubation, 41.4\%, 70\% and $95.8 \%$ of the initial insecticide dose were degraded by bacterial strain $\mathrm{P} 9$, respectively. Besides, P9 also shows the ability to use MP as the only carbon source (similar to the publication of Mansee et al. [14], Deng et al. [7]).

\subsubsection{Effect of the $\mathrm{pH}$}

After 7 days of shaking culture in MM medium containing MP $50 \mathrm{mg} / 1$ with different $\mathrm{pH}$ (pH5-9) (Figure 6) showed that: at $\mathrm{pH} 6$, the best growth $(\mathrm{OD} 600=1.28)$ and best MP reducing $(19.3 \mathrm{mg} / \mathrm{l}$ from initial concentration $50 \mathrm{mg} / \mathrm{l})$.
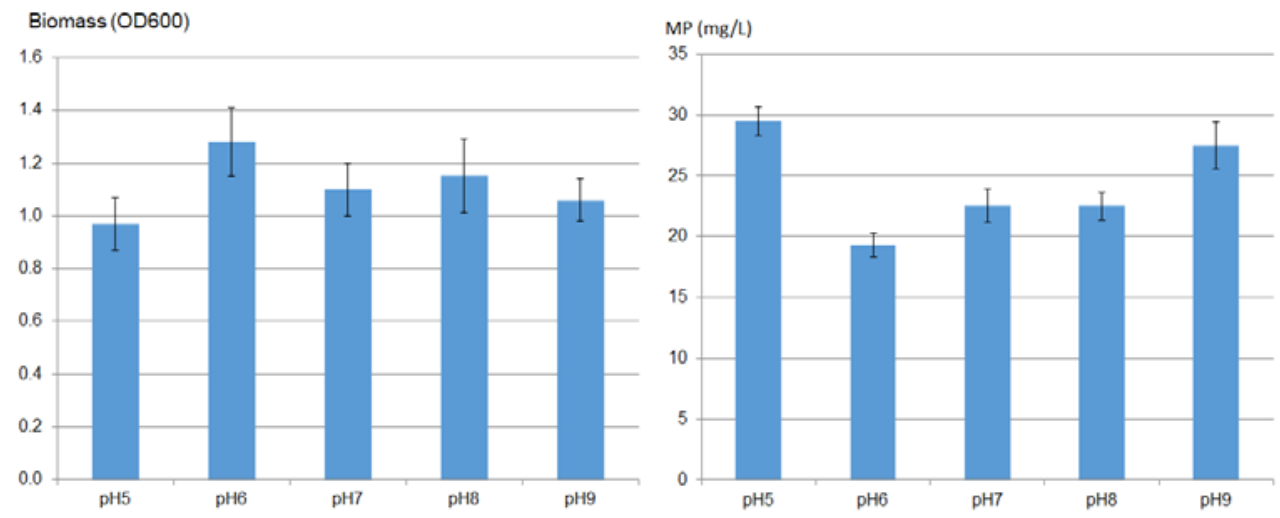

Fig. 6. Effect of the $\mathrm{pH}$ on the growth (left) and MP degradation (right)

\subsubsection{Effect of the medium}

P9 strain were cultured in MM, MMG (MM containing 0.5\% glucose), Wakimoto and LB media containing MP $50 \mathrm{mg} / 1$, after 7 days of culture to evaluate MP degradation ability by GC-MS.
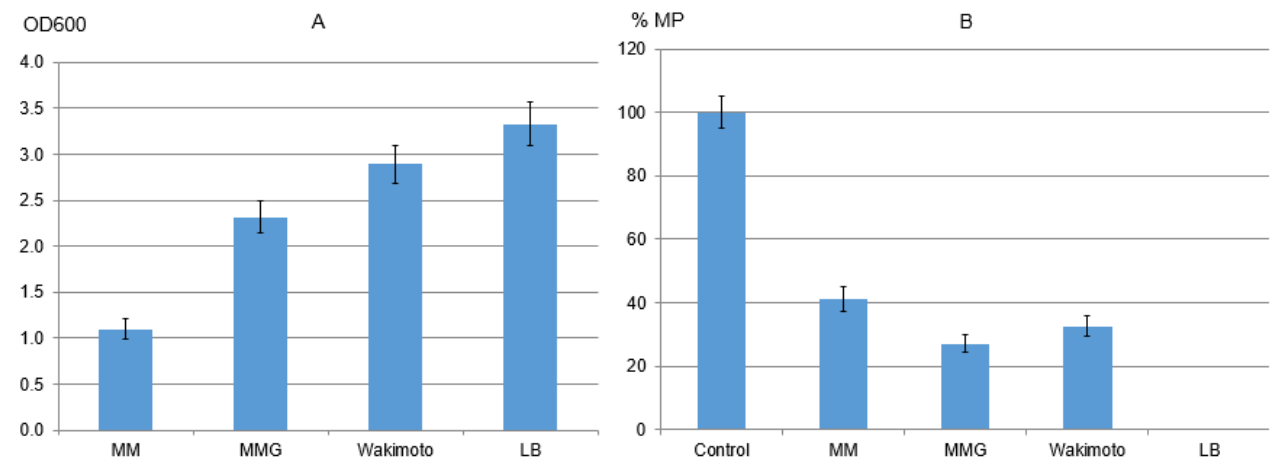

Fig. 7. Effect of the medium on the MP growth (A) and degradation (B)

The results obtained from the preliminary analysis of GC-MS are displayed in Figure 7. P9 strain grew best in LB medium, then Wakimoto, MMG, and MMG was the worst (Fig. 7A). MP degradation was best in LB medium, then MMG, Wakimoto and the worst in MM (Fig. 7B). P9 grew the strongest in LB medium $(\mathrm{OD} 600=3.3)$ and degradated all MP after 7 days. Chaudhry et al. published: when cultured in LB, both cells and crude cell extracts of P. diminuta and Pseudomonas sp. degraded MP and parathion well [9]. Wakimoto medium 
was also used by Verma et al. in strain screening and culture for good growth and organophosphorus hydrolase activity [15]. In these study cultures, MM gave the worst growth $(\mathrm{OD} 600=1.1)$. When supplementing with nutrients $0.5 \%$ glucose $(\mathrm{MMG})$, strain grew about 2 times stronger and MP degradation increased about 1.5 times. The results are consistent with previous studies: Bacterial growth on glucose-supplemented medium was 34 times (Mahiudddin et al.) [16] and 2 times (Cycon et al.) [17] that of the minimum medium in the first 2 days. Similar enhanced OP degradation in the presence of glucose in MSM was reported [16-17].

\subsubsection{Effect of the time}

The strains grew and reduced MP strongly on the first 6 days and grew most strongly after 6 days of culture (OD600=3.34) and degraded all MP50 mg/l after 7 days. After 2 days of incubation, the strain degraded 35.8\% MP (Fig. 8). Mansee et al. [14] also cultured, evaluate the ability to degrade MP with initial concentrations of $10-250 \mathrm{ppm}(10-250 \mathrm{mg} /$ 1) of 5 isolates D1-5 after 48 hours ( 2 days) of incubation show: all isolates reduced $30-70 \%$ MP at the initial MP $10 \mathrm{ppm}$. D1 and D2 degraded best over $40 \%$ at most concentrations. At initial concentration of 50 ppm, 3 strains D3-5 reduced about 20\% MP [14] (lower than this study). This result was consistent with the study of Mansee et al. [14] and which confirmed that lower degradation ability than our study. However our result were low compared with report of Deng et al. Stenotrophomonas sp. G1 degraded 100\% of MP 50 $\mathrm{mg} / \mathrm{l}$ in 24h [7] or Liu et al.: Acinetobacter radioresistens USTB-04 could completely degrade MP $1200 \mathrm{mg} / 1$ after 4 days [18].

Up to now, limited publication about Klebsiella ability of degrading OPs [3, 13] and our results shows the first time that Klebsiella variicola has published to degrade MP and Diazinon.

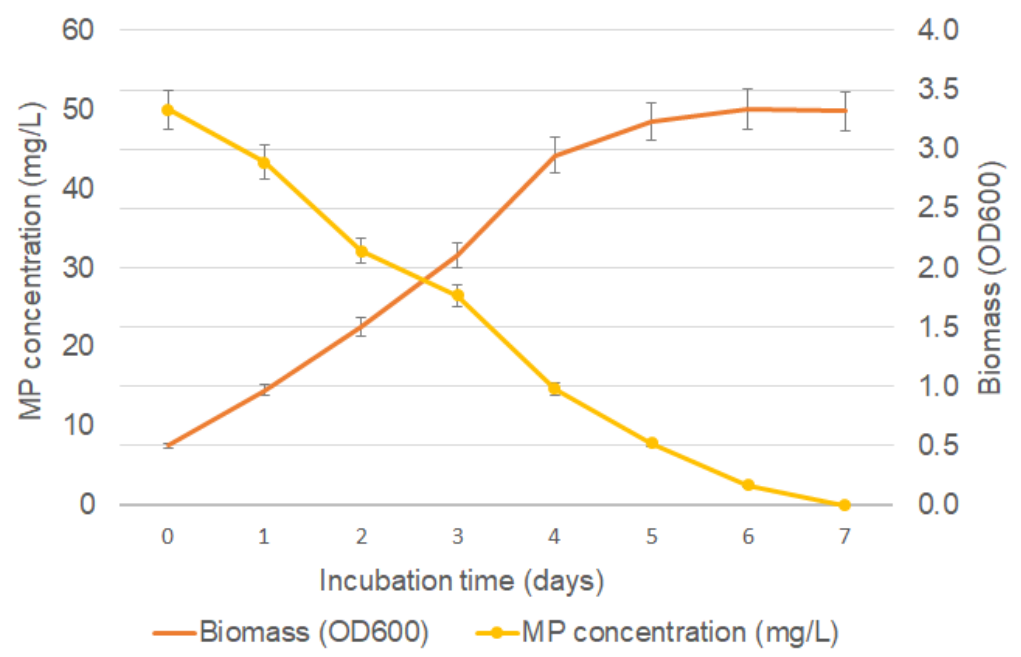

Fig. 8. Effect of the time on the MP degradation and growth of strain P9

\section{Conclusions}

These experiments confirmed that six OP-degrading strains were isolated from agricultural land of Ha Nam, Vietnam in which H14 (identified as Priestia aryabhattai) and P9 (identified as Klebsiella variicola) degraded best both MP and Diazinon. Use of these 
strains showing the potential for biological decontamination of the pesticide OPs and solving environmental pollution spots.

This research is supported by the basic project of Vietnam - Russia Tropical Center

\section{References}

1. K.E. LeJeune, B.C. Dravis, F. Yang, A.D. Hetro, B.P. Doctor, A.J. Russell, Ann N Y Acad Sci, 864, 153-70 (1998).

2. K. Alejo-González, E. Hanson-Viana, R. Vazquez-Duhalt, J Pestic Sci, 43(1), 1-9 (2018).

3. S. Kumar, G. Kaushik, M.A. Dar, S. Nimesh, U.J. Lopez-Chuken, and J.F., Pedosphere, 28(2), 190-208 (2018).

4. S. Ramu, B. Seetharaman, J Environ Sci Health B., 49(1): p. 23-34 (2014).

5. S. Pailan, D. Gupta, S. Apte, S. Krishnamurthi, P. Saha, Int Biodeterior. Biodegradation, 103, 191-195 (2015).

6. M. Cycoń, A. Żmijowska, M. Wójcik, Z. Piotrowska-Seget, J Environ Manage, 117, 7-16 (2013).

7. S. Deng, Y. Chen, D. Wang, T. Shi, X. Wu, X. Ma, X. Li, R. Hua, X. Tang, Q.X. Li, J Hazard Mater, 297, 17-24 (2015).

8. S.A.A. Firozjaei, A.M. Latifi, S. Khodi, S. Abolmaali, A. Choopani, J Appl Biotechnol Reports, 2(2), 215-224 (2015).

9. G.R. Chaudhry, A.N. Ali, W.B. Wheeler, Appl Environ Microbiol, 54(2), 288-93 (1988).

10. J. Sherma, W. Bretschneider, J Liq Chromatogr, 13(10), 1983-1989 (1990).

11. N. Sethunathan, T. Yoshida, Can J Microbiol, 19(7), 873-5 (1973).

12. C.S. Miller, K.M. Handley, K.C. Wrighton, K.R. Frischkorn, B.C. Thomas, J.F. Banfield, PloS one, 8(2), e56018 (2013).

13. S.V. Kulkarni, V.L. Markad, J.S. Melo, S.F. D'Souza, K.M. Kodam, Appl Microbiol Biotechnol, 98(2), 919-29 (2014).

14. A.M. Mansee, M.M.R. Montasser, R.A. Amer, Life Sci J, 14(10), 86-93 (2017).

15. N. Verma, J. Saini, S. Virk, M. Kataria, J Pharm Innov, 3(7), 71-76 (2014).

16. M. Mahiudddin, A.N.M. Fakhruddin, Abdullah-Al-Mahin, M.A.Z. Chowdhury, M.A. Rahman, M.K. Alam, Inter J Biotechnol, 3(1), 12-23 (2014).

17. M. Cycoń, M. Wójcik, Z. Piotrowska-Seget, Chemosphere, 76(4), 494-501 (2009).

18. F. Liu, M. Hong, D. Liu, Y. Li, P. Shou, H. Yan, G. Shi, J Environ Sci, 19(10), 1257-60 (2007). 